\title{
Inhibitory Effect of Oxethazaine on Midazolam Metabolism in Rats
}

\author{
Hokuto Namba, ${ }^{*, a}$ Yuki Nishimura, ${ }^{a}$ Norimitsu Kurata,${ }^{b}$ Mariko Iwase,${ }^{a}$ Takahito Hirai, ${ }^{a}$ and \\ Yuji Kiuchi ${ }^{a}$ \\ ${ }^{a}$ Department of Pharmacology, Showa University, School of Medicine; 1-5-8 Hatanodai, Shinagawa-ku, Tokyo 142- \\ 8555, Japan: and ${ }^{b}$ Faculty of Arts and Sciences at Fujiyoshida, Showa University; 4562 Kamiyoshida, Fujiyoshida, \\ Yamanashi 403-0005, Japan. \\ Received December 26, 2016; accepted June 18, 2017
}

There have been few reports concerning to the drug-drug interactions (DDIs) with OTC drugs although an increase in the use of OTC drugs in recent years. This current study was conducted to clarify the DDIs through CYP3A inhibition by oxethazaine (OXZ), an antacid available as an OTC drug. Midazolam (MDZ) was used as a probe drug for CYP3A activity. In an in vivo study, a single oral dose of OXZ $(50 \mathrm{mg} / \mathrm{kg}) \mathrm{was}$ administered to rats 30,60 , or $120 \mathrm{~min}$ before oral $\mathrm{MDZ}$ administration $(15 \mathrm{mg} / \mathrm{kg})$. Serum concentrations of $\mathrm{MDZ}$ were analyzed by HPLC, and its pharmacokinetic parameters were compared with a water-treated control group. The inhibitory effect of $\mathrm{OXZ}$ on MDZ 1'-hydroxylation (MDZ 1'-OH) activity was investigated in vitro using rat liver and intestinal microsomes. Pretreatment of OXZ $120 \mathrm{~min}$ before MDZ administration significantly increased the area under the serum concentration-time curve $\left(A U C_{0_{-\infty}}\right)$ of $M D Z$ six-fold compared to the control group without a change in elimination half-life $\left(t_{1 / 2}\right)$. In contrast, OXZ pretreatment 30 or $60 \mathrm{~min}$ before MDZ administration did not show any remarkable change in MDZ pharmacokinetic parameters. The in vitro study showed that $\mathrm{OXZ}$ inhibited MDZ 1'-OH activity in a concentration-dependent manner both in liver and intestinal microsomes. These results suggested that $\mathrm{OXZ}$ increases serum MDZ concentration presumably by the inhibition of liver and/or intestinal CYP3A activity. OXZ was predicted to cause the DDIs mediated by CYP3A inhibition, although this effect depended on the dose interval.

Key words OTC drug; oxethazaine; drug-drug interaction; CYP3A

OTC drugs can be easily purchased at the drugstore and on the internet without a doctor's prescription. In Japan, with the increase in the elderly population and the incidence of lifestyle-related diseases, self-medication is expected to become more widespread. The number of nonprescription OTC drugs that have been approved as prescription drugs has also increased. ${ }^{1,2)}$ For this reason, the use of OTC drugs is more likely to increase in the future and attract more attention in the clinics.

OTC drugs have been considered relatively safe for use. However, in recent years, diphenhydramine, an antihistamine that has been available on prescription drug and OTC for many years, was reported to inhibit CYP2D6-mediated metoprolol metabolism in humans. ${ }^{3)}$ Thus, the possibility of drug-drug interactions (DDIs) between diphenhydramine and CYP2D6 substrates with narrow therapeutic indices was suggested. Although most of OTC drugs have been available for a long time, information regarding their metabolic pathways and DDIs with other drugs is inadequate. The safety use of OTC drugs with respect to DDIs is therefore important.

Many gastrointestinal drugs can be easily obtained as OTC, and their high-use frequency increases the likelihood of combined use with other drugs. In a previous study, we examined the inhibitory effects of five OTC gastrointestinal drugs on CYP activities in human liver microsomes. The results showed that oxethazaine (OXZ) competitively inhibited CYP3A activity with a $K_{\mathrm{i}}$ value of $3.08 \mu \mathrm{m}$ (personal data). OXZ exerts a local anesthetic effect on gastric mucosa (Fig. 1). This drug inhibits gastric acid secretion secondarily by suppressing of gastrin secretion. ${ }^{4)}$ In fact, OXZ is used to alleviate pain associated with peptic ulcer disease, heartburn, and anorexia and is available OTC and on prescription in Asian countries such as Japan, Korea, and India. ${ }^{5,6)}$ Because OXZ is used to improve the common gastrointestinal symptoms, there may be multiple opportunities to take it in combination with various drugs: those used regularly for the maintenance treatment of comorbidities, or those taken temporarily for acute conditions such as the common cold. However, it has not been clarified whether DDIs through CYP3A inhibition occurs with OXZ in vivo as well as in vitro.

OXZ is usually taken after symptom onset. The timing of administration is often entrusted to the patient, so various inter-dose intervals are possible between concomitant drugs. Meanwhile, the pharmacologic effects of OXZ that raise gastric $\mathrm{pH}$ and inhibit gastrointestinal motility ${ }^{7,8)}$ may influence the pharmacokinetics of the concomitant drugs depending on the inter-dose interval. For this reason, it is necessary to investigate the DDI by OXZ at various inter-dose intervals rather than on co-administration with other drugs.

The current study was conducted to clarify the DDI through CYP3A inhibition by OXZ and investigate the inhibitory effect of OXZ on CYP3A-mediated midazolam (MDZ) metabolism in rats. The inter-dose intervals of both drugs were settled at $30,60,120$ min considering the actual situation<smiles>CN(C(=O)CN(CCO)CC(=O)N(C)C(C)(C)Cc1ccccc1)C(C)(C)Cc1ccccc1</smiles>

Fig. 1. Chemical Structure of Oxethazaine 
to take them.

\section{MATERIALS AND METHODS}

Chemicals MDZ maleate was a kind gift from Nippon Roche Co., Ltd. (Tokyo, Japan). OXZ and diazepam were obtained from Wako Pure Chemical Industries, Ltd. (Osaka, Japan). 1'-Hydroxymidazolam was purchased from Daiichi Pure Chemicals Co., Ltd. (Tokyo, Japan). All other chemicals were of analytical grade.

Animals Male Sprague-Dawley (SD) rats, weighing 220-240 g, were obtained from Japan Laboratory Animals Inc. (Tokyo, Japan). Rats were bred at an animal laboratory for one week as an adaptation period and starved overnight before in vivo studies.

The study procedure was reviewed and approved by the Showa University Ethics Committee for Animal Care and Use.

Treatment and Sampling Considering its pharmacological action, OXZ was predicted to combine with CYP3A substrates at various dosage intervals in different clinical situations. For this reason, we investigated the effect of OXZ treatment on serum MDZ concentrations at three different dose intervals. The dose of OXZ was determined based on the previous report that investigated the inhibition of gastric acid secretion in rats. ${ }^{7)}$

Rats were divided into four groups of 3-4 each. MDZ was orally administered to rats at a dose of $15 \mathrm{mg} / \mathrm{kg}$ after 30,60 , or $120 \mathrm{~min}$ of single oral treatment with OXZ $50 \mathrm{mg} / \mathrm{kg}$. Control rats were given water $30 \mathrm{~min}$ before $\mathrm{MDZ}$ administration.

Blood samples $(0.2 \mathrm{~mL})$ were collected from jugular vein before and after 15,30,45, 60,90, 120, 180, and $240 \mathrm{~min}$ of MDZ administration. The samples were centrifuged at $15000 \times \boldsymbol{g}$ for $10 \mathrm{~min}$ at $4^{\circ} \mathrm{C}$, and collected serum samples were stored at $-80^{\circ} \mathrm{C}$ until analysis.

Determination of Serum Midazolam Concentrations The serum concentrations of MDZ were determined using previously described HPLC methods with slight modifications. ${ }^{9-11)}$ Briefly, $100 \mu \mathrm{L}$ of each serum was diluted with $500 \mu \mathrm{L}$ of $0.1 \mathrm{~N} \mathrm{NaOH}$, and $5 \mathrm{ng}$ of diazepam was added as an internal standard. The mixture was extracted with $8 \mathrm{~mL}$ of dichloromethane-pentane $(1: 1)$ for $1 \mathrm{~min}$ and centrifuged at $1600 \times \boldsymbol{g}$ for $10 \mathrm{~min}$. The upper organic solvent phase was transferred into a clean glass tube and evaporated to dryness under the nitrogen stream. The dried residue was dissolved in $60 \mu \mathrm{L}$ of mobile phase, and $15 \mu \mathrm{L}$ was injected onto an HPLC equipped with a CAPCELLPAK C18 MG II column $(2.0 \times 250 \mathrm{~mm}$, and Shiseido, Tokyo, Japan). As the mobile phase 50:50 mixture (v/v) of $10 \mathrm{~mm}$ phosphate buffer $(\mathrm{pH} 5.0)$ $\mathrm{CH}_{3} \mathrm{CN}$ was used with the flow rate of $0.2 \mathrm{~mL} / \mathrm{min}$. UV detector was set at $220 \mathrm{~nm}$.

Pharmacokinetic Analysis The pharmacokinetic parameters of MDZ were assessed by non-compartment analysis using MOMENT $\left(\right.$ Excel $\left.^{\circledR}\right)$ based on the moment analytic method. ${ }^{12,13)}$ The area under the serum concentration-time curve $\left(A U C_{0-\infty}\right)$ was calculated according to the trapezoidal rule. The maximum serum concentrations $\left(C_{\max }\right)$ was obtained from the actual data. The elimination half-life $\left(t_{1 / 2}\right)$ was calculated, dividing $\ln 2$ by $\lambda$, which is the terminal elimination rate constant calculated by a linear regression analysis of four or more data points from the terminal portion.

Assay of CYP3A Activity Pooled liver and intestinal mi- crosomes were prepared from male SD rats according to the conventional methods ${ }^{14-16)}$ to investigate the inhibitory effect of OXZ on MDZ 1'-hydroxylation (MDZ 1'-OH) activity in vitro. All microsomes were frozen by liquid nitrogen and kept at $-80^{\circ} \mathrm{C}$ until used. Protein concentrations were measured by the method of Lowry et al. ${ }^{17}$

The reaction mixtures consisted of pooled rat liver $(100 \mu \mathrm{g})$ or intestinal $(200 \mu \mathrm{g})$ microsomes, $30 \mu \mathrm{M} \mathrm{MDZ}$, and the nicotinamide adenine dinucleotide phosphate (NADPH) generating system in $80 \mathrm{~mm}$ phosphate buffer $(\mathrm{pH}$ 7.4) were incubated at $37^{\circ} \mathrm{C}$ for $15 \mathrm{~min}$ in the presence of $\mathrm{OXZ}$ at the final concentration of $1-300 \mu \mathrm{M}$. The reactions were quenched by the addition of cold methanol-acetonitrile $(35: 21)$, and then nitrazepam (200 ng) was added as an internal standard. 1'-hydroxymidazolam was analyzed by HPLC according to a previous report. ${ }^{18)}$ Control activity was determined in the same conditions but with the addition of water instead of OXZ. The analytic results were shown as the percentage of control activity.

In addition, the preincubation study was conducted to determine the possibility of the metabolite or metabolic intermediate production of OXZ that inhibit or inactivate CYP3A. OXZ $(3 \mu \mathrm{M})$ was preincubated with pooled rat liver microsomes at $37^{\circ} \mathrm{C}$ for 10,20 , and $30 \mathrm{~min}$ in the presence or absence of NADPH. After the preincubation step, MDZ was added and MDZ 1'-OH activity was analyzed as described above.

Statistical Analysis In vivo data were presented as the mean \pm standard deviation (S.D.). The differences between the four groups were analyzed by non-repeated measures ANOVA followed by the Bonferroni's method. A difference of $p<0.05$ was considered statistically significant.

\section{RESULTS}

Pharmacokinetics of MDZ The serum concentrationtime profiles and the pharmacokinetic parameters of MDZ administered orally after 30,60 , or $120 \mathrm{~min}$ of oral OXZ treatment are shown in Fig. 2 and Table 1.

In the group treated with OXZ $120 \mathrm{~min}$ before MDZ administration, the mean $A U C_{0-\infty}$ and $C_{\text {max }}$ of MDZ increased from $710 \pm 255$ to $4562 \pm 690 \mathrm{ng} \cdot \mathrm{h} / \mathrm{mL}$ and from $615 \pm 192$ to $3083 \pm 486 \mathrm{ng} / \mathrm{mL}$, respectively ( $p<0.01$ versus controls), without a change in $t_{1 / 2}$. Administration of OXZ 30 or $60 \mathrm{~min}$ before MDZ administration showed a tendency toward an increase in MDZ $A U C_{0-\infty}$ and $C_{\max }$ but the differences were not statistically significant.

MDZ 1'-Hydroxylation Activity in Vitro OXZ inhibited CYP3A-mediated MDZ 1'-OH activity in a concentrationdependent manner both in rat liver and intestinal microsomes with $\mathrm{IC}_{50}$ values of 8.6 and $65 \mu \mathrm{M}$, respectively (Fig. 3).

To identify the possible contribution of CYP-mediated metabolites or active intermediates on the inhibitory effect of OXZ against CYP3A activity, another inhibition study was conducted including a preincubation step. As a result, the increase of inhibition potency of OXZ on MDZ 1'-OH activity was not observed even after $30 \mathrm{~min}$ of preincubation (Fig. 4).

\section{DISCUSSION}

The current study was conducted to clarify the DDIs between the OTC antacid OXZ and MDZ through CYP3A 


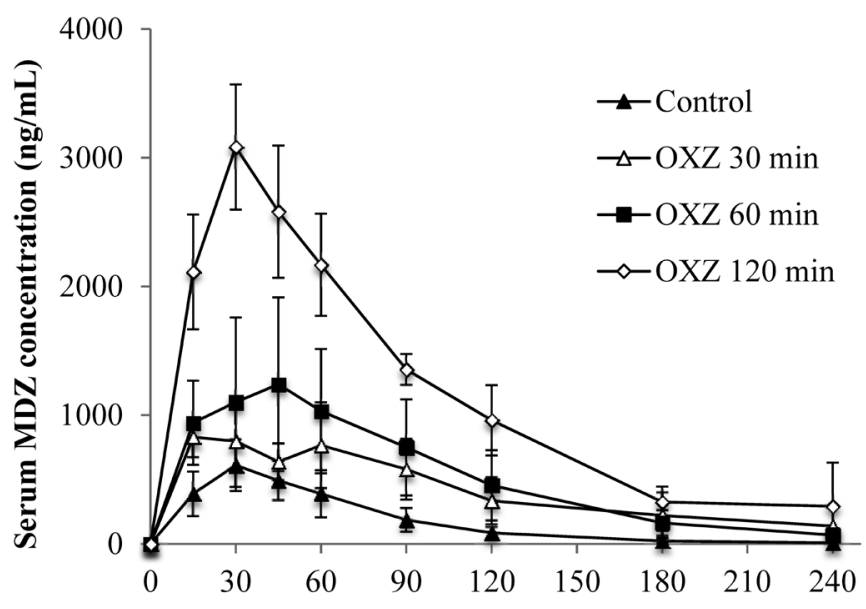

Time after MDZ administration (min)

Fig. 2. Serum Concentration-Time Profiles of Midazolam (MDZ) after a Single Treatment of Oxethazaine (OXZ) in Rats

OXZ $(50 \mathrm{mg} / \mathrm{kg})$ was orally administered to rats, followed by oral administration of $\mathrm{MDZ}(15 \mathrm{mg} / \mathrm{kg})$ after 30,60 , or $120 \mathrm{~min}$. Control rats were treated with water $30 \mathrm{~min}$ before $\mathrm{MDZ}$ administration. Each point represents the mean \pm S.D. of three to four rats.

Table 1. Pharmacokinetic Parameters of Midazolam (MDZ) after a Single Treatment of Oxethazaine (OXZ) in Rats

\begin{tabular}{ccccc}
\hline \hline $\begin{array}{c}\text { Dose interval } \\
(\mathrm{min})\end{array}$ & & $\begin{array}{c}A U C_{0-\infty} \\
(\mathrm{ng} \cdot \mathrm{h} / \mathrm{mL})\end{array}$ & $\begin{array}{c}C_{\max } \\
(\mathrm{ng} / \mathrm{mL})\end{array}$ & $\begin{array}{c}t_{1 / 2} \\
(\mathrm{~min})\end{array}$ \\
\hline 30 & Control & $710 \pm 255$ & $615 \pm 192$ & $33.11 \pm 4.21$ \\
& OXZ & $2090 \pm 1062$ & $1083 \pm 162$ & $76.84 \pm 43.98$ \\
60 & $\mathrm{OXZ}$ & $2191 \pm 1066$ & $1445 \pm 683$ & $44.23 \pm 10.77$ \\
120 & $\mathrm{OXZ}$ & $4562 \pm 690^{*}$ & $3083 \pm 486^{*}$ & $31.10 \pm 6.80$ \\
\hline
\end{tabular}

Data are shown as the mean \pm S.D. of $3-4$ rats. ${ }^{*} p<0.01$ versus control.

inhibition. Our results revealed that OXZ inhibited MDZ metabolism in rats.

Our previous in vitro study has shown that OXZ inhibited MDZ 1'-OH activity in human liver microsomes (personal data). In the current study, the concentration-related inhibition of MDZ 1'-OH activity by $\mathrm{OXZ}$ was also observed in intestinal microsomes as well as liver microsomes in rats. Furthermore, this in vivo study showed that pretreatment with OXZ changed MDZ pharmacokinetic parameters, and the degree of this effect differed according to the dose interval between the two drugs. When OXZ was given $120 \mathrm{~min}$ before MDZ administration, MDZ $A U C_{0-\infty}$ and $C_{\max }$ showed a remarkable five-to-six-fold increase compared to the control group, without a change in $t_{1 / 2}$. These results suggested that OXZ significantly increased serum MDZ concentrations presumably by the inhibition of liver and/or intestinal CYP3A activity. Generally, in addition to CYPs, drug transporters such as P-glycoprotein (P-gp) have an important role in the pharmacokinetics of orally administered drugs. ${ }^{19)}$ Therefore, if the intestinal absorption by P-gp was inhibited by OXZ, blood concentrations of the concomitant drug increase. However, because MDZ is not a substrate of P-gp, ${ }^{19)}$ this possibility can be excluded.

At the same time, it is known that OXZ is mainly metabolized in liver with a short $t_{1 / 2}$ of $1 \mathrm{~h}$, and less than $0.1 \%$ of the unchanged form is excreted in the urine within $24 \mathrm{~h}^{20)}$ Never-

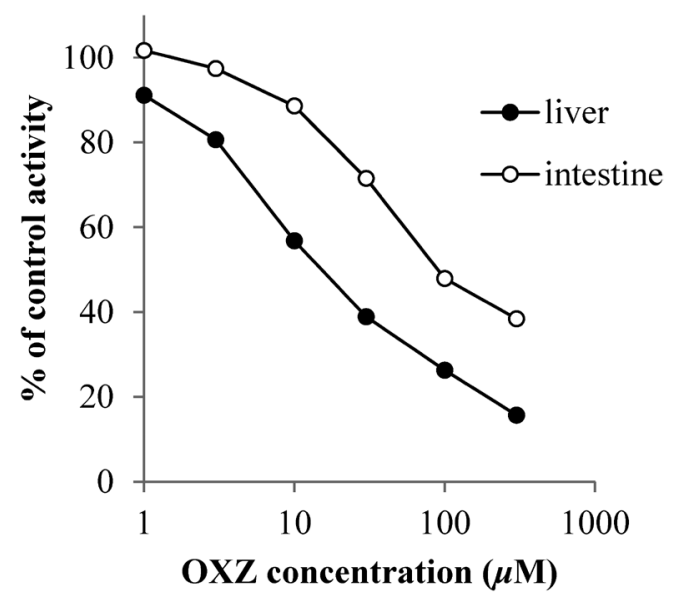

Fig. 3. Inhibitory Effect of Oxethazaine (OXZ) on Midazolam 1'-Hydroxylation (MDZ 1'-OH) Activity in Rat Liver and Intestinal Microsomes

OXZ $(1-300 \mu \mathrm{M})$ was incubated in the incubation mixture including rat liver or intestinal microsomes, MDZ $(30 \mu \mathrm{M})$ and nicotinamide adenine dinucleotide phosphate (NADPH). Data are shown as the mean of duplicate determinations.

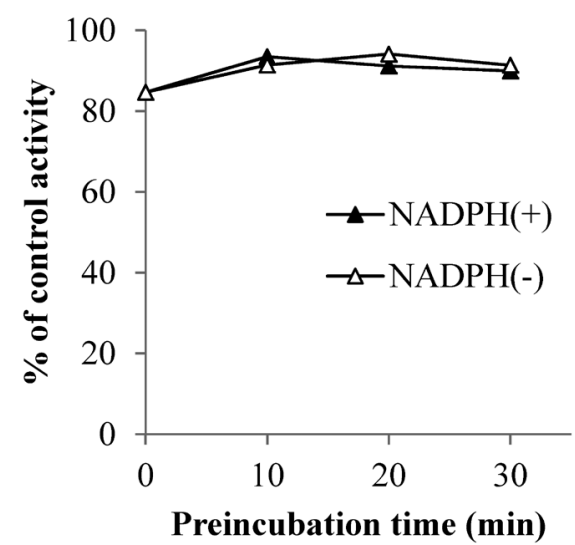

Fig. 4. Preincubation Effect of Oxethazaine (OXZ) on Midazolam 1'-Hydroxylation (MDZ 1'-OH) Activity in Rat Liver Microsomes

OXZ $(30 \mu \mathrm{M})$ was preincubated with rat liver microsomes for 10, 20, and $30 \mathrm{~min}$ in the presence or absence of nicotinamide adenine dinucleotide phosphate (NADPH) before the addition of MDZ. Data are shown as the mean of duplicate determinations.

theless, the pharmacokinetics of OXZ has not been clarified enough and the amount of OXZ remaining in liver or small intestine $120 \mathrm{~min}$ after its oral administration is unknown. For the DDI study mediated by intestinal CYP3A, as the maximal concentration of inhibitors in the intestinal lumen after oral administration, it is recommended to use dose $/ 250 \mathrm{~mL} .^{21}$ ) According to this, the predicted value of maximal intestinal concentration of $\mathrm{OXZ}$ is estimated to reach higher enough compared to $\mathrm{IC}_{50}$ value of $65 \mu \mathrm{M}$ obtained by an in vitro experiment. Thus, OXZ is thought to have likelihood to inhibit intestinal CYP3A activity even $120 \mathrm{~min}$ after oral administration.

Meanwhile, little information is available concerning OXZ metabolism, only that mephentermine and phentermine are known to be its major metabolites. ${ }^{5)}$ Particularly, the involvement of CYPs on OXZ metabolism has not been reported. However, because it is known that phentermine does not inhibit CYP3A4 activity ${ }^{22}$ and also mephentermine is further metabolized to phentermine, these metabolites are not likely 
to inhibit CYP3A activity. In addition, as one of the mechanism of CYP inhibition, a mechanism-based inhibition (MBI) has been demonstrated. In this case, the inhibitory effect persists because the reactive intermediate that is catalytically activated by CYP binds to a target CYP irreversibly. Therefore, we conducted an in vitro that included a preincubation step to clarify the possibility of OXZ as a mechanism-based inhibitor. As a result, no additional inhibition of MDZ 1'-OH was observed when $\mathrm{OXZ}$ was preincubated with rat liver microsomes and NADPH before the addition of MDZ. Therefore, the inhibition mechanism of OXZ on CYP3A activity did not seem to exert a MBI, which the intermediate metabolite of OXZ inhibited this enzyme irreversibly. These results suggested that the inhibitory effect of OXZ itself on CYP3A was involved in the marked elevation of serum MDZ level.

In contrast to the remarkable effect of $\mathrm{OXZ}$ on serum $\mathrm{MDZ}$ concentration with a dose interval of $120 \mathrm{~min}$, OXZ administration 30 or 60 min before MDZ treatment showed no significant change in the pharmacokinetic parameters of MDZ. One possible explanation for the different influence of OXZ treatment on MDZ metabolism at different dose intervals is the pharmacological effect of OXZ on MDZ. Because OXZ has the ability to restrain gastrointestinal motility that reaches the maximum from 30 to $60 \mathrm{~min}$ after dosage. ${ }^{23)}$ Therefore, MDZ might have been transferred to the small intestine gradually when OXZ was given 30 or $60 \mathrm{~min}$ before MDZ administration. For this reason, it was suggested that rapid increase of serum MDZ concentration did not occur even if intestinal CYP3A was inhibited by OXZ treatment. In fact, the serum MDZ concentration-time profiles with a broad apex were observed in the 30 or 60 min dose interval group as shown in Fig. 2.

In general, because $\mathrm{OXZ}$ is used for the improvement of gastrointestinal symptoms, patients do not take this drug before symptom onset. Accordingly, it is speculated that patients might take OXZ at various dose intervals, including $120 \mathrm{~min}$ after taking other drugs. Therefore, the investigation of DDIs, including the consideration of inter-dose intervals, can provide more practical information for clinical situations.

OXZ is as available on prescription and OTC. Despite their easy availability, there has been little information about DDIs between OTC drugs: therefore, the doctors and pharmacists are unable to give the appropriate safety warnings to patients. As shown in the current study, it is notable that $\mathrm{OXZ}$ which is used as OTC drug increased the $A U C_{0-\infty}$ of the concomitant drug six-fold. The cause of this DDI was not proved directly because we could not measure the concentration of OXZ in the liver and small intestine and did not examine the inhibitory effect of OXZ treatment on intravenously administered MDZ concentration. Nevertheless, MDZ is known to be a typical probe drug to estimate CYP3A-mediated metabolism and many drugs are metabolized via CYP3A. Because gastrointestinal medications such as $\mathrm{OXZ}$ are more likely to be used together with other drugs, careful attention is necessary to avoid severe DDIs. The DDIs observed in this study on rats suggests the need for a clinical study on humans. Furthermore, more studies investigating DDIs between other OTC drugs are warranted.

In the current study, we have shown that OXZ markedly increased serum MDZ concentration in rats. This DDI was probably caused by the mechanism including liver and/or intestinal
CYP3A inhibition. The effect of OXZ treatment on MDZ metabolism differed according to dose interval, as shown by the significant six-fold increase in the $A U C_{0-\infty}$ of $\mathrm{MDZ}$ when $\mathrm{OXZ}$ was administered $120 \mathrm{~min}$ before MDZ treatment.

Conflict of Interest The authors declare no conflict of interest.

\section{REFERENCES}

1) Nomura K, Kitagawa Y, Yuda Y, Takano-Ohmuro H. Medicine reclassification processes and regulations for proper use of over-thecounter self-care medicines in Japan. Risk Manag. Healthc. Policy, 9, 173-183 (2016).

2) Chang J, Lizer A, Patel I, Bhatia D, Tan X, Balkrishnan R. Prescription to over-the-counter switches in the United States. J. Res. Pharm. Pract., 5, 149-154 (2016).

3) Hamelin BA, Bouayad A, Methot J, Jobin J, Desgagnes P, Poirier P, Allaire J, Dumesnil J, Turgeon J. Significant interaction between the nonprescription antihistamine diphenhydramine and the CYP2D6 substrate metoprolol in healthy men with high or low CYP2D6 activity. Clin. Pharmacol. Ther., 67, 466-477 (2000).

4) Yasuno R, Oguma T, Masuda Y. $\mathrm{Ca}^{2+}$ enhancement of hemolysis induced by the topical anesthetic oxethazaine in vitro. Biol. Pharm. Bull., 21, 1294-1299 (1998).

5) Lee SY, You IJ, Kim MJ, Kwon SH, Hong SI, Kim JH, Jang MH, Oh SM, Chung KH, Lee SY, Jang CG. The abuse potential of oxethazaine: effects of oxethazaine on drug-seeking behavior and analysis of its metabolites in plasma and hair in animal models. Pharmacol. Biochem. Behav., 105, 98-104 (2013).

6) Bhoir S, Bhagwat AM. Comparison of seven oxethazaine containing antacids available in the Indian market. J. Assoc. Physicians India, 61, 400-403 (2013).

7) Narikawa H, Oyama R, Harigaya S. Direct action of timepidium bromide on the gastric mucosa and its inhibitory effect on pepsin secretion. Nippon Yakurigaku Zasshi, 78, 71-78 (1981).

8) Jankelson OM, Jankelson IR. The symptomatic, exaggerated gastrocolic reflex: its relief by an oral, topical anesthetic (oxethazaine hydrochloride). Am. J. Gastroenterol., 32, 719-722 (1959).

9) Saito $Y$, Nishimura $Y$, Kurata N, Iwase M, Aoki K, Yasuhara H. In vivo inhibition of CYP3A-mediated midazolam metabolism by anchusan on rats. J. Pharmacol. Sci., 115, 399-407 (2011).

10) Mandema JW, Tukker E, Danhof M. Pharmacokinetic-pharmacodynamic modeling of the EEG effects of midazolam in individual rats: influence of rate and route of administration. Br. J. Pharmacol., 102, 663-668 (1991).

11) Toshima H, Sanbe T, Kizaki J, Oguchi K, Uchida N, Nishimura Y, Iwase M, Harimoto Y, Kawakami M, Hirosawa M, Kobayashi S. Inhibition of CYP3A4-mediated midazolam metabolism by repeat oral administration of anchusan in humans. Showa Gakushikaishi, 73, 120-128 (2013).

12) Tabata K, Yamaoka K, Kaibara A, Suzuki S, Terakawa M, Hata T. Moment analysis program available on Microsoft Excel ${ }^{(\mathbb{B}}$. Drug Metab. Pharmacokinet., 14, 286-293 (1999).

13) Yamaoka K, Tanigawara $Y$, Nakagawa T, Uno T. A pharmacokinetic analysis program (multi) for microcomputer. J. Pharmacobiodyn., 4 , 879-885 (1981)

14) Guengerich FP. Analysis and characterization of enzymes. Principles and methods of toxicology. (Hayes AW ed.) Third edition, Raven Press, New York, pp. 1259-1313 (1994).

15) Bonkovsky HL, Hauri HP, Marti U, Gasser R, Meyer UA. Cytochrome P450 of small intestinal epithelial cells. Immunochemical characterization of the increase in cytochrome P450 caused by phenobarbital. Gastroenterology, 88, 458-467 (1985).

16) Koudriakova T, Iatsimirskaia E, Tulebaev S, Spetie D, Utkin I, 
Mullet D, Thompson T, Vouros P, Gerber N. In vivo disposition and metabolism by liver and enterocyte microsomes of the antitubercular drug rifabutin in rats. J. Pharmacol. Exp. Ther., 279, 1300-1309 (1996).

17) Lowry OH, Rosebrough NJ, Farr AL, Randall RJ. Protein measurement with the Folin phenol reagent. J. Biol. Chem., 193, 265-275 (1951).

18) Nishimura Y, Kurata N, Sakurai E, Yasuhara H. Inhibitory effect of antituberculosis drugs on human cytochrome P450-mediated activities. J. Pharmacol. Sci., 96, 293-300 (2004).

19) Kim RB, Wandel C, Leake B, Cvetkovic M, Fromm MF, Dempsey PJ, Roden MM, Belas F, Chaudhary AK, Roden DM, Wood AJ, Wilkinson GR. Interrelationship between substrates and inhibitors of human CYP3A and P-glycoprotein. Pharm. Res., 16, 408-414 (1999).
20) Hsu MC, Lin SF, Kuan CP, Chu WL, Chan KH, Chang-Chien GP. Oxethazaine as the source of mephentermine and phentermine in athlete's urine. Forensic Sci. Int., 185, e1-e5 (2009).

21) The Ministry of Health. Labor and Welfare of Japan. "Drug interaction guideline for drug development and labeling recommendations (draft for public comment) (2014).”: 〈http://www.solvobiotech.com/ documents/Japanese_DDI_guideline_(draft)_2014Jan.pdf), cited 20 March, 2017.

22) Center for Drug Evaluation and Research, Food and Drug Administration. "Clinical Pharmacology and Biopharmaceutics Review(s).": 〈http://www.accessdata.fda.gov/drugsatfda_docs/nda/2012/ 022580Orig1s000ClinPharmR.pdf), cited 20 March, 2017.

23) Tamaki H, Meshi T. Relationship between pharmacological activity and blood level of a new anticholinergic agent, timepidium bromide (SA-504), in cats. Jpn. J. Pharmacol., 26, 209-215 (1976). 